\title{
1 Rapid and efficient generation of antigen-specific isogenic T cells from
}

2

3

4 5

6

7

8

9

10

11

12

13

14

15

16

17

18

19

20

21

22

23

24

25

26

27

28

29

30

31

\section{cryopreserved blood samples}

AL Eerkens $^{1,2}$, A Vledder $^{1,2}, \mathrm{~N}$ van Rooij ${ }^{1}$, F Foijer $^{3}$, HW Nijman $^{1,4}$, M de Bruyn ${ }^{1,4}$

1 University of Groningen, University Medical Center Groningen, Department of Obstetrics and Gynecology, The Netherlands.

${ }^{2}$ Contributed equally

${ }^{3}$ University of Groningen, University Medical Center Groningen, European Research Institute for the Biology of Ageing, The Netherlands.

${ }^{4}$ Shared senior authorship

Corresponding author:

Dr. M. de Bruyn

University Medical Center Groningen

CMC V, 4e floor room Y4.240

PO 30.001

9700 RB Groningen

Tel ${ }^{+} 31(0) 503613174$

$\mathrm{Fax}^{+} 31(0) 503611806$

Emailm.de.bruyn@umcg.nl

Word Count: 1933

Abstract: 243

Tables/Figures: 4

References: 23 
1

\section{ABSTRACT}

Objectives CRISPR/Cas9-mediated gene editing has been leveraged for the modification of human and mouse T cells. However, limited experience is available on the application of CRISPR/Cas9 electroporation in cryopreserved T cells collected during e.g. clinical trials.

Methods PBMCs from healthy donors were used to generate knockout T cell models for interferon- $\nu$ (IFNY), Cbl Proto-Oncogene B (CBLB), Fas cell surface death receptor (Fas) and T cell receptor (TCR $\alpha \beta b$ ) genes. The effect of CRISPR-cas9-mediated gene editing on T cells was evaluated using apoptosis assays, cytokine bead arrays and ex vivo and in vitro stimulation assays.

Results Our results demonstrate that CRISPR/Cas9-mediated gene editing of ex vivo T cells is efficient and does not overtly affect T cell viability. Cytokine release and T cell proliferation were not affected in gene edited T cells. Interestingly, memory T cells were more susceptible to CRISPR/Cas9 gene editing than naïve T cells. Ex vivo and in vitro stimulation with antigens resulted in equivalent antigen-specific

T cell responses in gene-edited and untouched control cells; making CRISPR/Cas9-mediated gene editing compatible with clinical antigen-specific T cell activation and expansion assays.

Conclusion Here, we report an optimized protocol for rapid, viable and highly efficient genetic modification in ex vivo human antigen specific T cells, for subsequent functional evaluation and/or expansion. Our platform extends CRISPR/Cas9-mediated gene editing for use in gold-standard clinically-used immune-monitoring pipelines and serves as a starting point for development of analogous approaches such as those including transcriptional activators and or epigenetic modifiers. 


\section{INTRODUCTION}

CRISPR/Cas9-mediated gene targeting has been used to significantly improve our understanding of T cell biology and discover regulators of e.g. T cell proliferation and differentiation (1-3). To date, most of this work has been performed using cultured peripheral blood mononuclear cells (PBMCs) from healthy donors, or using patient T cells after an initial ex vivo expansion (3). In this setting, cell numbers are not limited and there is generally no need to maintain the clonal repertoire of T cells. By contrast, samples obtained within clinical trials in immune oncology are generally limited and antigen-specificity paramount in understanding the underlying immune biology (4-6). Expanding the CRISPR/Cas9 toolbox to antigen-specific T cells from clinical samples would therefore enable further dissection of anti-cancer immune responses and discovery of novel targets for combination immunotherapy (1).

Herein, special consideration should be given to maintaining the ex vivo T cells' phenotype and function during the process of gene engineering $(4,7)$. In addition, a CRISPR/Cas9-mediated gene targeting protocol for clinical samples should ideally be compatible with the timelines used in bestpractice immunomonitoring protocols. As such, viral-mediated transfection models, such as lentivirus, adenovirus and retrovirus with variable knockout efficiencies and T cell toxicity are unsuitable (8-10). Ribonucleoprotein (RNP) complexes of Cas9 and a guide RNA (gRNA), when introduced by electroporation, have been reported as suitable for ex vivo modification of T cells, but whether a highlevel of viability and clonality can be maintained for incorporation into immune monitoring protocols remains unclear $(8,9)$.

Here, we report on an optimized protocol for rapid, viable, and highly efficient genomic manipulation in ex vivo human antigen-specific T cells. We demonstrate the efficacy of this gene editing system within common immunomonitoring pipelines and across gRNAs. This system is robust across donors and useable for gene editing in both memory and naïve T cells for studying recall and de novo responses, respectively. 


\section{RESULTS}

\section{CRISPR/Cas9-mediated gene editing of ex vivo T cells does not overtly affect T cell viability}

We set out to optimize a CRISPR/Cas9-mediated gene editing protocol compatible with PBMC samples analogous to those routinely procured during clinical trials. PBMCs were processed according to best practice operating protocols for PBMC isolation, cryopreservation and cell density used in storage. Using these standardized PBMC aliquots, we first optimized RNP complex electroporation using efficiency and viability as main parameters. As proof-of-concept, we targeted IFNY as it is not expressed ex vivo in T cells, readily induced upon activation and easily assessable by ELISA or intracellular flow cytometry. Electroporation of Cas9/IFNY-gRNA RNP complexes into T cells resulted in almost complete abrogation of IFNy expression in both CD8 and CD4 T cells (figure 1a, c). Electroporation, inclusion of Cas9/gRNA complexes and/or crRNA alone, had no effect on T cell viability as measured using amine-reactive dye staining (figure 1b, e). By varying experimental parameters, we observed that while Cas9 protein concentration did not affect $T$ cell viability, the size of the electroporation cuvette was critically important, resulting in a reduced relative viability when using $20 \mu \mathrm{L}$ cuvettes compared to $100 \mu \mathrm{L}$ cuvettes (figure 1d). IFNy knockout efficiency was similar for all experimental conditions and in both CD4 and CD8 T cells (figure 1d). As lower doses of Cas9 protein were equally effective, we chose 100 $\mu \mathrm{g} / \mathrm{mL}$ in $100 \mu \mathrm{L}$ cuvettes as optimal concentration in all subsequent experiments. Analysis of additional parameters of cell viability: 7-AAD, Phosphatidyl Serine (PS) exposure and caspase activation confirmed the minimal loss of cell viability under these conditions (figure 1e). Non-T cells in the PBMC fraction were more sensitive to the T cell optimized protocol, as evident from increased cell death in the total PBMC fraction (figure 1f).

\section{CRISPR/Cas9-mediated gene editing of ex vivo T cells does not overtly affect T cell function}

Next, we tested whether our optimized protocol affected $T$ cell function by evaluating cytokine production and proliferation of gene edited T cells. While release of IFNY into the extracellular space was markedly reduced in IFNy knockout T cells, no differences were observed in the release of other cytokines, such as TNF $\alpha$ and IL-2 (figure 2a, b, supplementary figure 1). Atypical induction of IL-4, IL-5 or IL-10 were also not observed under these conditions (figure $2 \mathrm{a}, \mathrm{b}$ ). We also analyzed whether ex vivo gene engineered $T$ cells required recovery following electroporation by activating $T$ cells using antiCD3/CD28 beads at $0,30,60,120$ and 240 minutes after RNP nucleofection, followed by 2 day culture and restimulation using PMA/ionomycin. Under all conditions, production of IFNy, but not TNF $\alpha$ or IL-2, was specifically abrogated (figure 2c, 2d). Accordingly, anti-CD3/CD28 bead- or PHA-based expansion of $T$ cells directly following electroporation did not impair expansion capacity over a period of 14 days (figure 2e). Furthermore, gene-edited T cells that expanded over this period remained IFNY-negative, suggesting the optimized protocol did not negatively affect $T$ cell fitness when compared with unedited 
T cells (figure $2 \mathrm{f}$ and supplemental figure 1 ). We reasoned that the direct ex vivo gene editing, followed

115 by immediate $T$ cell activation, could even be leveraged to knockout genes critical for anti-CD3/CD28 bead-based activation, as the steady-state protein levels in the absence of transcription are likely sufficient to drive signaling during the initial 24-48 hours of culture. To test this hypothesis, we disrupted expression of the T cell receptor (TCR $\alpha \beta$ ) complex. T cells were activated using anti-CD3/CD28 beads directly after RNP electroporation. Again, CRISPR Cas9/TCR $\alpha \beta$-gRNA RNP complex electroporation resulted in high TCR $\alpha \beta$ knockout efficiency in both CD8 and CD4 T cells at $48 \mathrm{~h}$ (figure 2g). As anticipated, TCR $\alpha \beta$ knockout T cells proliferated comparably to non-edited T cells over a period of 14 days (figure 2h). As for IFNY, no detrimal effects of gene editing were observed and near-complete loss of TCR $\alpha \beta$ was maintained at day 14 (figure 2i).

We next determined whether memory or naïve T cells were more amenable to CRISPR/Cas9-mediated gene editing in the ex vivo setting. PBMCs were subjected to TCR $\alpha \beta$-targeting RNP electroporation, followed by 8-day culture with cytokines IL-2, IL-15 and/or IL-7 to support memory and naïve T cells, respectively. Cell viability was similar across all conditions (figure 3a). Percentages of memory CD8 T cells were similar between TCR $\alpha \beta$ knockout and TCR $\alpha \beta$-expressing cells regardless of the cytokine cocktail used (figure 3b,c). Notably, there was a slight increase in the percentage of memory CD4 T cells after TCR $\alpha \beta$ knockout compared to the TCR $\alpha \beta$-proficient cells, independent of cytokine stimulation (figure 3c).

To further explore this observation, we investigated the knockout efficiency within the memory and naïve T cell populations (figure 3d-i). The knockout efficiency of memory T cells varied between 6085\%, in $\mathrm{CD}^{+} \mathrm{T}$ cells and between $70-95 \%$ in $\mathrm{CD}^{+} \mathrm{T}$ cells (figure $3 \mathrm{~d}, \mathrm{~g}$ ). By contrast, the knockout efficiency for naïve T cells ranged between $35-80 \%$ in CD8 T cells and between $30-80 \%$ in CD4 T cells (figure 3e,h). In addition, the percentage of naïve TCR $\alpha \beta$ knockout CD8 T cells was higher when compared to naïve TCR $\alpha \beta$ knockout CD4 T cells (figure 3e,h). These findings collectively suggest that memory T cells, especially memory CD4 T cells, are slightly more susceptible to CRISPR/Cas9 gene editing than naïve T cells.

CRISPR/Cas9-mediated gene editing is compatible with clinical antigen-specific T cell activation and

\section{4 expansion assays}

145 Finally, having established the high viability and efficacy of our approach, we evaluated whether our 146 optimized protocol was compatible with best-practice assays for monitoring immune responses in 147 clinical trial settings. We analyzed ex vivo peptide stimulation, as well as in vitro stimulation (IVS) 148 assays. Using CEF peptides as model antigen, and T cell activation markers CD69 and CD137 as a 
149 readout, we were able to demonstrate equivalent CEF-induced responses in gene-edited and

150 untouched control cells for both the ex vivo assay (figure 4a, c) and following IVS (figure 4b, d). These

151 responses were observed for IFNy knockouts, as well as for Cbl Proto-Oncogene B (CBLB) and Fas cell

152 surface death receptor (Fas) gRNAs previously reported by others (figure 4a-d).

153

154 


\section{DISCUSSION}

156

157

158

159

160

161

162

163

164

165

166

167

168

169

170

171

172

173

174

175

176

177

178

179

180

181

182

183

184

185

186

187

We describe a rapid, robust and flexible platform for CRISPR/Cas9-mediated gene editing in primary ex vivo human T cells. We have optimized efficiency, viability, as well as culture conditions to allow rapid and direct ex vivo modification of $T$ cells for subsequent functional evaluation and/or expansion. Our platform is amenable to integration into clinically used pipelines for immune response evaluation using antigens of choice.

CRISPR/Cas9-mediated gene editing has become an attractive approach for modifying T cells, due to its simplicity, operability, low costs and capability of multiplex genome editing $(9,11)$. However, to date, limited experience on the application of CRISPR/Cas9 electroporation in immune monitoring assays in clinical studies is available. The prerequisites for manipulating T cells in immunoassays are maintaining viability, antigen-specificity and function $(4,7)$. In this study, we demonstrate for the first time that these prerequisites are not affected by CRISPR/Cas9 gene editing, thereby providing a proofof-principle for the application of CRISPR/Cas9 in immune monitoring assays. In both CD8 and CD4 T cells, a highly efficient gene knock-out up to $90 \%$ was reached after a single transfection. Previously published literature, using CRISPR/cas9 RNP electroporation, reported efficiency rates of $20-90 \%$ with low efficiency rates in resting primary T cells $(9,12-16)$. Moreover, we demonstrated that CRISPR/Cas9edited cells could be maintained without stimulation in culture for up to 7 days, or supplemented with a diverse range of cytokines without comprising gene knockout efficacy. Activation of T cells directly, or at a later time-point of choosing did not negatively affect $T$ cell function, expansion, nor did cryopreservation and subsequent restimulation. Finally, while the current work focused on knockout of genes, recent advances in non-homologous end joining (NHEJ)-mediated template incorporating using CRISPR/cas9 should be amenable to the current platform, allowing more complex ex vivo $\mathrm{T}$ cell engineering $(12,14,17)$.

Advances in descriptive immune monitoring have expanded our understanding of anti-cancer immune responses. High-parameter flow, mass and spectral cytometry have allowed the simultaneous assessment of most immune cell populations ex vivo $(18,19)$. Imaging using e.g. PET/SPECT allows noninvasive assessment of specific immune cells or immune checkpoints throughout the body, and transcriptomic approaches provide ever deeper dimensional assessment of gene expression, including spatial organization (20-22). By contrast, ex vivo functional assays have remained largely unchanged over the years, with peptide stimulation and intracellular flow cytometry, ELISPOT or ELISA used for functional readout on one or two hallmark activation markers $(4,23)$. The platform we present here significantly expands the potential of these assays. By seamlessly integrating CRISPR/Cas9-mediated gene editing into ex vivo immune monitoring pipelines, it is possible to assess how T cells from clinically- 
treated patients differentially depend on genes-of-interest, but also screen ex vivo for novel

189 combination targets to further augment $T$ cell activation $(14,15)$. As demonstrated here, these targets

190 need not be limited to cell surface targets accessible by antibody-based therapeutics $(14,15)$. One

191 interesting observation is that naïve CD4, but not CD8, T cells appear less sensitive to CRISPR/Cas9-

192 mediated gene editing than their memory counterparts. Whether this is the result of the optimized

193 electroporation protocol used, or an intrinsic feature of naïve CD4 T cells is currently unknown.

194 Nevertheless, targeted genes of interest could be knocked out in 60\% of CD4 T cells, establishing our

195 platform as an effective approach for studying both recall and de novo immune responses. An

196 interesting approach would be to further combine our platform with recent advances in CRISPR/Cas9-

197 mediated gene editing in monocyte-derived dendritic cells. This could allow reciprocal knockout of

198 ligand-receptor interactions of particular interest for complex ligand-receptor pairs, such as CTLA-

$1994 / C D 28$ and CD80/CD86.

200 Taken together, our platform represents an advance on previous reports on CRISPR/Cas9-

201 mediated gene editing and extends this approach to use in gold-standard clinically-used immune-

202 monitoring pipelines. 


\section{MATRIALS AND METHODS}

206

207

208

209

210

211

212

213

214

215

216

217

218

219

220

221

222

223

224

225

226

227

228

229

230

231

232

233

234

\section{Peripheral Blood Mononuclear Cells}

Peripheral blood mononuclear cells (PBMCs) were isolated from blood of healthy volunteers after written informed consent was obtained (Sanquin). For isolation, buffy coats were mixed at a 1:2 ratio with RPMI supplemented with 2,5\% FCS, layered on a Ficoll-Paque gradient at a 1:2 volume ratio and centrifuged at $900 \mathrm{~g}$ for 20 minutes without brake. PBMC on the interface of the Ficoll-Paque and plasma layers were isolated by pipette $(5-10 \mathrm{~mL})$, supplemented with ice-cold PBS to a final volume of $50 \mathrm{~mL}$ and centrifuged at $560 \mathrm{~g}$ for 8 minutes without brake. PBMC pellets were pooled, supplemented with ice-cold PBS to a final volume of $50 \mathrm{~mL}$ and centrifuged at $350 \mathrm{~g}$ for 8 minutes without brake. After PBMCs were resuspended in $50 \mathrm{~mL}$ of ice-cold PBS, PBMCs were counted using the Bürker counting chamber and centrifuged at $350 \mathrm{~g}$ at 8 minutes without brake. Finally, PBMCs were resuspended in 1 $\mathrm{mL}$ of freezing medium, consisting of $90 \% \mathrm{FCS}$ and $10 \%$ DMSO, at a concentration of $10-100^{6}$ cells per cryovial.

\section{Cas9, tracRNA and crRNA}

Cas9 (Alt-R Cas 9 Nuclease V3), tracRNA (Alt-R CRISPR-Cas9 tracRNA) and the crRNAs (Alt-R CRISPR-Cas9 crRNA) were synthesized by Integrated DNA Technologies (Coralville, USA). Each crRNA was reconstituted in nuclease free duplex buffer (Lonza) to a final concentration of $100 \mu \mathrm{M}$. See Table 1 for gRNAs and sequences. For TCR $\alpha$ : equal volumes of TRAC1 and TRBC were mixed before adding the RNP complex to the PBMCs, see RNP electroporation.

\section{RNP electroporation}

PBMCs were counted and resuspended in X-VIVO $15^{\mathrm{TM}}$ at densities as indicated. After optimization as indicated, the final protocol to prepare Cas9/gRNA RNP complexes was as follows: 150 pmol annealed gRNA per nucleofection condition was prepared by combining $1.5 \mu \mathrm{L}$ tracRNA with $1.5 \mu \mathrm{L}$ crRNA. The mix was annealed by heating at $95^{\circ} \mathrm{C}$ for 5 minutes and slowly cooled down to room temperature for 10 minutes. Next, $12 \mu \mathrm{L}$ duplex buffer (Lonza, Basel, Switzerland) was added to get a final concentration $150 \mathrm{pmol}$ gRNA in $15 \mu \mathrm{l}$. To prepare a Cas9/gRNA RNP complex, $10 \mu \mathrm{g}$ Cas9 $(10 \mu \mathrm{g} / \mathrm{ml})$ was added to the gRNA complex. This mixture was incubated for 10 minutes at room temperature. Finally, duplex buffer was added to the RNP complex to obtain a final volume of $20 \mu \mathrm{L}$.

For electroporation, PBMCs were centrifuged and washed once with PBS. Cells were resuspended in $100 \mu \mathrm{L}$ nucleofection solution at densities as indicated. Nucleofection solution consisted of $82 \mu \mathrm{L}$ P2 Primary Cell Nucleofector ${ }^{\mathrm{TM}}$ Solution (Lonza) and $18 \mu \mathrm{L}$ Supplement 1 (Lonza, Basel, Switzerland) for $100 \mu \mathrm{L}$ cuvettes and $16.4 \mu \mathrm{L}$ P2 Primary Cell Nucleofector ${ }^{\mathrm{TM}}$ Solution and $3.6 \mu \mathrm{L}$ Supplement 1 for $20 \mu \mathrm{L}$ cuvettes. RNP complex was added to the PBMCs and incubated for 2 minutes 
at room temperature. The PBMC/RNP mixture was transferred to a nucleofection cuvette, followed by electroporation of cells using a 4D-Nucleofector machine (Amaxa, program P2, pulse code EH100). PBMCs that did not undergo electroporation served as control and were centrifuged and washed with PBS.

\section{$\underline{\text { Intracellular cytokine staining }}$}

16 hours after electroporation, PBMCs were stimulated for 4 hours with PMA/lonomycin (eBioscience stimulation cocktail) at $37^{\circ}$ degrees. For intracellular staining of IFNץ, tumor necrosis factor alpha (TNF $\alpha$ ), and interleukin-2 (IL-2), secretion of cytokines was blocked using golgiplug (protein transport inhibitor; BD 555029). After 4 hours, cells were stained with Zombie Aqua viability dye according to manufacturer's protocol and washed with PBS supplemented with 5\% FCS. Next, cells were fixed and permeabilized (CEL FIXATIE \& PERMEABILISATIE KIT, GAS-002, Nordic-Mubio) and stained with surface markers CD3, CD8, CD4, and intracellular markers IFNY and IL-2 for flow cytometer analysis. For the TCR $\alpha \beta$ knockout experiments, only cell surface staining with TCR $\alpha \beta, C D 3, C D 8$ and CD4 antibodies was performed. See supplementary table 2 for characteristics of the antibodies. For fixation and permeabilization cells were incubated with $100 \mu \mathrm{L} /$ well of reagent A (fix) for 15 minutes at room temperature. Next, cells were washed with PBS and incubated with a mixture of reagent B (perm) and antibodies for 15 minutes at room temperature. In total $100 \mu \mathrm{L}$ of the mixture reagent B plus antibodies was added per well. After incubation, cells were washed twice, centrifuged and resuspended in flow cytometry buffer (PBS + 2\% FCS).

Intracellular staining of IFN with anti-CD3/CD28 beads (Thermofisher, Cat. 11131D) at 0, 30, 60, 120 and 240 minutes after RNP nucleofection, followed by 2 day culture and restimulation using PMA/ionomycin.

\section{Apoptosis assay}

Apoptosis was assessed 24 hours after CRISPR/Cas9-mediated gene editing. PBMCs were counted, centrifuged and resuspended in X-VIVO $15^{\mathrm{TM}}$ at a density of $1-2 \times 10^{6}$ cells $/ \mathrm{mL}$. Subsequently, $1 \mu \mathrm{L}$ Violet live Caspase probe per $0.5 \mathrm{~mL}$ of cell suspension was added and cells were incubated for 45 minutes at $37^{\circ} \mathrm{C}$. After washing, cells were resuspended in fresh medium and incubated for an additional 30 minutes at $37^{\circ} \mathrm{C}$ while protected from light. Afterwards, CD8 and CD3 antibodies were added and cells were incubated for 30 minutes at room temperature. Cells were washed twice with cold PBS and resuspended in $1 \times$ Annexin $\vee$ Binding Buffer at a final concentration of $1 \times 10^{6}$ cells $/ \mathrm{mL}$. $1 \times 10^{5}$ cells were incubated with $5 \mu \mathrm{L}$ of Annexin $V$ and $5 \mu \mathrm{L}$ of 7-Aminoactinomycin $D$ Invitrogen ${ }^{\mathrm{TM}}$ (7-AAD) for 15 minutes at room temperature in the dark. Cells were analyzed within 1 hour using the BD FACSVerse flow cytometer (BD Biosciences). 
Cytokine bead array

276 One day after electroporation $\left(10 \times 10^{6}\right.$ cells in $2 \mathrm{~mL}, 1 \mathrm{~mL}$ per well), gene-edited and control PBMCs 277 were incubated with PMA/ionomycin (eBioscience stimulation cocktail) for 4 hours at $37 C^{\circ}$. 278 Subsequently, supernatant was collected, and stored at $-80^{\circ} \mathrm{C}$.

279 The human Th1/Th2 Cytokine Assay (BD ${ }^{\text {TM }}$ Cytometric Bead Array (CBA) Human Th1/Th2 Cytokine Kit II) was used to quantitatively measure cytokine production according to the manufacturer's protocol. In brief, supernatant was thawed and $50 \mu \mathrm{L}$ was used for the cytokine bead array. Samples and standards were incubated with the capture beads for 3 hours at room temperature, while protected from light (Supplementary data, supplementary table 2). After incubation, assay tubes were washed, centrifuged and resuspended before flow cytometer analysis.

\section{T cell expansion assay}

PBMCs were plated at a density of $5 \times 10^{6} / \mathrm{ml}$ in a 24 wells plate. Expansion of gene-edited PBMCs was tested by stimulation with CD3/CD28 beads ( $20 \mu \mathrm{L}$ per well, $5 \times 10^{6}$ cells per well) or phytohemagglutinin (L1668 Sigma)(PHA, $10 \mu \mathrm{g} / \mathrm{mL}$ ). IL-2 (50 IU/mL) was added to both stimulating conditions. Stimulation was done directly after electroporation. Plates were incubated at $37^{\circ} \mathrm{C}$ and cells were counted and at day 7, 10 and 14 . Cell density was maintained around $1 \times 10^{6}$ cells $/ \mathrm{mL}$ and medium, IL-2 and/or PHA was refreshed at day 7 , and 10 .

\section{Ex vivo T cell stimulation}

PBMCs were resuspended in $500 \mu \mathrm{L}$ culture medium (X-VIVO $15^{\mathrm{TM}}$ with $10 \%$ human serum) and plated in $100 \mu \mathrm{L} /$ well of 96 -well round bottom plates (Thermofisher) at a density of $1 \times 10^{6} / \mathrm{ml}$. Cells were ex vivo stimulated either with viral peptides consisting of a pool of 23 different peptides originating from Cytomegalovirus, Epstein-Barr virus and influenza virus peptides (CEF) or with interleukin 7 (IL-7) and interleukin 15 (IL-15). CEF was synthesized by JPT Peptide Technology (Berlin, Germany). Recombinant human IL-7 (Asp26-His177, size $10 \mu \mathrm{g}$ ) and recombinant human IL-15 (amino acids Asn49-Ser162, size $10 \mu \mathrm{g})$ were purchased from Biolegend.

For ex vivo stimulation with $\mathrm{CEF}$, cells were activated one day after electroporation by incubation with CEF peptides at a concentration of $1 \mu \mathrm{g} / \mathrm{ml}$ at $37^{\circ} \mathrm{C}$. For ex vivo stimulation with IL-7 and IL-15, cells were activated by incubation with IL-7 and IL-15 at a concentration of $25 \mathrm{ng} / \mathrm{ml}$ at $37^{\circ} \mathrm{C}$ at day 8. After 16-26 hours of incubation with CEF or IL-7 and IL-15, cells were stained for T cell phenotype with CD3, CD8 and CD14 and for T cell activation with CD69 and CD137, see supplementary table 2. 
309 PBMCs were plated in $2 \mathrm{~mL}$ culture medium per well per CRISPR condition of 24-wells plates at a density

310 of $5 \times 10^{6} / \mathrm{ml}$. Culture medium composed of X-VIVO $15^{\mathrm{TM}}$ enriched with $10 \%$ human serum, $1 \%$

311 penicillin/streptomycin (P/S), 1\% L-glutamine (L-glu) and IL-2 (20 IU/ml). PBMCs were stimulated with

312 CEF for 13 days. A concentration of $1 \mu \mathrm{g} / \mathrm{mL}$ CEF was used. Medium was changed at day 4, 6, 8 and 11,

313 according to the harmonization protocol of short-term in vitro culture for expansion of antigen-specific

314 CD8+T cells (4). At day 13, cells were re-stimulated with CEF at a concentration of $1 \mu \mathrm{g} / \mathrm{mL}$ overnight at

$31537^{\circ} \mathrm{C}$ and $5 \% \mathrm{CO}_{2}$, while medium served as a negative control and PHA (L1668 Sigma) at $1 \mu \mathrm{g} / \mathrm{mL}$ as

316 positive control. After 16-26 hours cells were stained for phenotype with CD3 and CD8 and for activation

317 with CD69 and CD137, see supplementary table 2 for characteristics of the antibodies.

319 Memory T cells assay

320 PBMCs were resuspended in $800 \mu \mathrm{L}$ culture medium (X-VIVO $15^{\mathrm{TM}}$ with $10 \%$ human serum) and plated

321 in $100 \mu \mathrm{L} /$ well of 96 -well round bottom plates (Thermofisher) at a density of $0,625 \times 10^{6} / \mathrm{mL}$. PBMCs were

322 stimulated with IL-2, IL-7 and/or IL-15 at a concentration of $25 \mathrm{ng} / \mathrm{mL}$ or left untreated as indicated.

323 Cells were cultured for 7 days. At day 8, cells were stained for T cell memory phenotype with CD4, CD8,

$324 \mathrm{CD} 45$ and TCR $\alpha / \beta$, see supplementary table 2 for characteristics of the antibodies. 
Declaration of Interest statements:

327 FF Outside the submitted work, dr. Foijer reports grants from the Dutch Cancer Society (KWF)

328 HWN, Outside the submitted work, prof. Nijman reports grants from the Dutch Cancer Society (KWF),

329 grants from the European Research Council (ERC), grants from Health Holland, non-financial support

330 from AIMM Therapeutics, grants from Immunicum, non-financial support from BioNTech, non-financial

331 support from Surflay, grants and shares and non-financial support from Vicinivax; In addition, prof.

332 Nijman has grants and non-financial support from Aduro Biotech, in part relating to a patent for

333 Antibodies targeting CD103 (de Bruyn et al. No. 62/704,258).

$334 \mathrm{MB}$, Outside the submitted work, dr. de Bruyn reports grants from the Dutch Cancer Society (KWF),

335 grants from the European Research Council (ERC), grants from Health Holland, grants from Immunicum,

336 non-financial support from BioNTech, non-financial support from Surflay, grants and non-financial

337 support from Vicinivax; non-financial support from AIMM therapeutics; In addition, dr. de Bruyn has

338 grants and non-financial support from Aduro Biotech, in part relating to a patent for Antibodies targeting

339 CD103 (de Bruyn et al. No. 62/704,258).

340 There are no conflicts of interest to disclose for the remaining authors. 


\section{REFERENCES}

343 1. Liu D, Zhao X, Tang A, Xu X, Liu S, Zha L, et al. CRISPR screen in mechanism and target discovery for cancer immunotherapy. Biochim Biophys Acta - Rev Cancer [Internet]. 2020;1874(1):188378. Available from: https://doi.org/10.1016/j.bbcan.2020.188378

2. Henriksson J, Chen X, Gomes T, Ullah U, Meyer KB, Miragaia R, et al. Genome-wide CRISPR Screens in T Helper Cells Reveal Pervasive Crosstalk between Activation and Differentiation. Cell. 2019;176(4):882-896.e18.

3. Shifrut E, Carnevale J, Tobin V, Roth TL, Woo JM, Bui CT, et al. Genome-wide CRISPR Screens in Primary Human T Cells Reveal Key Regulators of Immune Function. Cell [Internet]. 2018;175(7):1958-1971.e15. Available from: https://doi.org/10.1016/j.cell.2018.10.024

4. Chudley L, McCann KJ, Coleman A, Cazaly AM, Bidmon N, Britten CM, et al. Harmonisation of short-term in vitro culture for the expansion of antigen-specific CD8+ T cells with detection by ELISPOT and HLA-multimer staining. Cancer Immunol Immunother. 2014;63(11):1199-211.

5. Dey S, Kamil Reza K, Wuethrich A, Korbie D, Ibn Sina AA, Trau M. Tracking antigen specific Tcells: Technological advancement and limitations. Biotechnol Adv. 2019;37(1):145-53.

6. Santegoets SJAM, Welters MJP, van der Burg SH. Monitoring of the immune dysfunction in cancer patients. Vaccines. 2016;4(3):1-23.

7. Lin Y, Gallardo HF, Ku GY, Li H, Manukian G, Rasalan TS, et al. Optimization and validation of a robust human T-cell culture method for monitoring phenotypic and polyfunctional antigenspecific CD4 and CD8 T-cell responses. Cytotherapy. 2009;11(7):912-22.

8. Oh SA, Seki A, Rutz S. Ribonucleoprotein Transfection for CRISPR/Cas9-Mediated Gene Knockout in Primary T Cells. Curr Protoc Immunol. 2019;124(1):1-18.

9. Seki A, Rutz S. Optimized RNP transfection for highly efficient CRI SPR/Cas9-mediated gene knockout in primary T cells. J Exp Med. 2018;215(3):985-97.

10. Roth TL, Puig-Saus C, Yu R, Shifrut E, Carnevale J, Li PJ, et al. Reprogramming human T cell function and specificity with non-viral genome targeting. Nature [Internet]. 2018;559(7714):405-9. Available from: http://dx.doi.org/10.1038/s41586-018-0326-5 Biotechnol. 2014;32(4):347-55. 
371 12. Hendel A, Bak RO, Clark JT, Kennedy AB, Ryan DE, Roy S, et al. Chemically modified guide RNAs enhance CRISPR-Cas genome editing in human primary cells. Nat Biotechnol. 2015;33(9):9859.

374

21. Zheng C, Zheng L, Yoo JK, Guo H, Zhang Y, Guo X, et al. Landscape of Infiltrating T Cells in Liver

13. Mandal PK, Ferreira LMR, Collins R, Meissner TB, Boutwell CL, Friesen M, et al. Efficient ablation of genes in human hematopoietic stem and effector cells using CRISPR/Cas9. Cell Stem Cell [Internet]. 2014;15(5):643-52. Available from: http://dx.doi.org/10.1016/j.stem.2014.10.004

14. Schumann K, Lin S, Boyer E, Simeonov DR, Subramaniam M, Gate RE, et al. Generation of knock-in primary human T cells using Cas9 ribonucleoproteins. Proc Natl Acad Sci U S A. 2015;112(33):10437-42.

15. Rupp LJ, Schumann K, Roybal KT, Gate RE, Ye CJ, Lim WA, et al. CRISPR/Cas9-mediated PD-1 disruption enhances anti-Tumor efficacy of human chimeric antigen receptor T cells. Sci Rep. $2017 ; 7(1): 1-10$.

16. Su S, Hu B, Shao J, Shen B, Du J, Du Y, et al. CRISPR-Cas9 mediated efficient PD-1 disruption on human primary T cells from cancer patients. Sci Rep. 2016;6:1-13.

17. Banan M. Recent advances in CRISPR/Cas9-mediated knock-ins in mammalian cells. J Biotechnol [Internet]. 2020;308(August 2019):1-9. Available from: https://doi.org/10.1016/j.jbiotec.2019.11.010

18. Pitoiset F, Cassard L, El Soufi K, Boselli L, Grivel J, Roux A, et al. Deep phenotyping of immune cell populations by optimized and standardized flow cytometry analyses. Cytom Part A. 2018;93(8):793-802.

19. Abdelaal T, van Unen V, Höllt T, Koning F, Reinders MJT, Mahfouz A. Predicting Cell Populations in Single Cell Mass Cytometry Data. Cytom Part A. 2019;95(7):769-81.

20. van de Donk PP, de Ruijter LK, Lub-De Hooge MN, Brouwers AH, van der Wekken AJ, Oosting SF, et al. Molecular imaging biomarkers for immune checkpoint inhibitor therapy. Theranostics. 2020;10(4):1708-18. Cancer Revealed by Single-Cell Sequencing. Cell [Internet]. 2017;169(7):1342-1356.e16. Available from: http://dx.doi.org/10.1016/j.cell.2017.05.035 Diverse Immune Phenotypes in the Breast Tumor Microenvironment. Cell [Internet]. 
403 23. Letsch A, Scheibenbogen C. Quantification and characterization of specific T-cells by antigenspecific cytokine production using ELISPOT assay or intracellular cytokine staining. Methods. 2003;31(2):143-9. 


\section{Supplementary table 1}

\begin{tabular}{|l|l|}
\hline gRNA & Sequence \\
\hline \hline IFNY & AAA GAG TGT GGA GAC CAT CA \\
\hline CBLB & TGCACAGAACTATCGTACCA \\
\hline Fas & GAGGGTCCAGATGCCCAGCA \\
\hline TCR $\alpha$ & TGTGCTAGACATGAGGTCTA \\
\hline TCR $\beta$ & GGAGAATGACGAGTGGACCC \\
\hline
\end{tabular}

\section{Supplementary table 2}

\begin{tabular}{|c|c|c|c|c|}
\hline Antibody & Color & Cat No & Supplier & Experiment \\
\hline CD3 & APC-eFluor 780 & $47-0038-42$ & $\begin{array}{l}\text { eBioscience }^{\mathrm{TM}} \text {, } \\
\text { ThermoFisher }\end{array}$ & $\begin{array}{l}\text { Ex vivo and in vitro T cell } \\
\text { stimulation }\end{array}$ \\
\hline CD3 & $\mathrm{PE}$ & $12-0038-41 / 42$ & $\begin{array}{l}\text { eBioscience }^{\mathrm{TM}} \\
\text { ThermoFisher }\end{array}$ & Apoptosis assay \\
\hline CD3 & FITC & 555332 & $\mathrm{BD}$ & Intracellular cytokine staining \\
\hline CD4 & PE & $12-0048-42$ & $\begin{array}{l}\text { eBioscience }^{\mathrm{TM}} \text {, } \\
\text { ThermoFisher }\end{array}$ & $\begin{array}{l}\text { Intracellular cytokine staining } \\
\text { Memory } T \text { cell assay }\end{array}$ \\
\hline CD8 & BV421 & 562428 & $\begin{array}{l}\text { eBioscience }^{\mathrm{TM}}, \\
\text { ThermoFisher }\end{array}$ & $\begin{array}{l}\text { Intracellular cytokine staining } \\
\text { Ex vivo and in vitro T cell } \\
\text { stimulation } \\
\text { Memory T cell assay }\end{array}$ \\
\hline CD8 & APC-eFluor 780 & $47-0088-42$ & $\begin{array}{l}\text { eBioscience }^{\mathrm{TM}} \text {, } \\
\text { ThermoFisher }\end{array}$ & $\begin{array}{l}\text { Apoptosis assay } \\
\text { Intracellular cytokine staining }\end{array}$ \\
\hline CD45RA & Pe-Cy7 & $25-0458-41$ & $\begin{array}{l}\text { eBioscience }^{\mathrm{TM}}, \\
\text { ThermoFisher }\end{array}$ & Memory T cell assay \\
\hline CD69 & FITC & $11-0699-41$ & $\begin{array}{l}\text { eBioscience }^{\mathrm{TM}} \\
\text { ThermoFisher }\end{array}$ & $\begin{array}{l}\text { Ex vivo and in vitro T cell } \\
\text { stimulation }\end{array}$ \\
\hline CD137 & $\mathrm{PE}$ & $12-1379-42$ & $\begin{array}{l}\text { eBioscience }^{\mathrm{TM}} \\
\text { ThermoFisher }\end{array}$ & $\begin{array}{l}\text { Ex vivo and in vitro T cell } \\
\text { stimulation }\end{array}$ \\
\hline $\mathrm{TCR} \alpha / \beta$ & APC & $17-9986-41$ & $\begin{array}{l}\text { eBioscience }^{\mathrm{TM}}, \\
\text { ThermoFisher }\end{array}$ & $\begin{array}{l}\text { Memory T cell assay } \\
\text { Intracellular cytokine staining }\end{array}$ \\
\hline IFNY & PerCP-Cy5.5 & $45-7319-42$ & $\begin{array}{l}\text { eBioscience }^{\mathrm{TM}}, \\
\text { ThermoFisher }\end{array}$ & Intracellular cytokine staining \\
\hline TNFa & APC & $17-7349-82$ & $\begin{array}{l}\text { eBioscience }^{\mathrm{TM}} \text {, } \\
\text { ThermoFisher }\end{array}$ & Intracellular cytokine staining \\
\hline $\mathrm{IL}-2$ & PE-Cy7 & $25-7029-42$ & $\begin{array}{l}\text { eBioscience }^{\mathrm{TM}}, \\
\text { ThermoFisher }\end{array}$ & Intracellular cytokine staining \\
\hline
\end{tabular}


Supplementary table 3

418

\begin{tabular}{|l|l|l|}
\hline Tube label & Concentration $(\mathrm{pg} / \mathrm{mL})$ & Cytokine Standard dilution \\
\hline \hline 1 & 0 (negative control) & No standard dilution (Assay Diluent only) \\
\hline 2 & 20 & $1: 256$ \\
\hline 3 & 40 & $1: 128$ \\
\hline 4 & 80 & $1: 64$ \\
\hline 5 & 156 & $1: 32$ \\
\hline 6 & 312,5 & $1: 16$ \\
\hline 7 & 625 & $1: 8$ \\
\hline 8 & 1250 & $1: 4$ \\
\hline 9 & 2500 & $1: 2$ \\
\hline 10 & 5000 & Top Standard \\
\hline
\end{tabular}




\section{FIGURE DESCRIPTIONS}

\section{Figure 1. CRISPR/Cas9 electroporation ex vivo T cells does not affect T cell viability}

Electroporation of PBMCs with Cas9/IFNy gRNA RNP complexes results in almost complete abrogation of IFNy expression in both CD8 and CD4 T cells. 16 hours after electroporation, PBMCs were stimulated for 4 hours with PMA/Ionomycin. (A, C) Representative flow cytometry contour plot showing the expression of IFNy production in CD8 T cells. (B) Scatterplots demonstrating the viability of PBMCs after electroporation alone, with Cas9/gRNA complexes and/or crRNA alone. Viability was measured using amine reactive-dye. (D) Effect of different cuvette sizes (100 and 20 $\mu \mathrm{L}$ ) and different Cas9 protein concentrations (0, 100, 250 en $500 \mu \mathrm{g} / \mathrm{ml}$ ) on cell viability. (E) Analysis of additional parameters of cell viability was performed using 7-AAD, Phosphatidyl Serine (PS) exposure and caspase activation in CD8 and CD4 T cells(E) and in the total PBMC fraction(F).

Blue dots represent unstimulated PBMCs; orange dots represent PBMCs stimulated with PMA/ionomycin for 4 hours; green dots represent all PBMCs; in e, middle scatterpot, blue dots represent CD8 T cells and orange dots CD4 T cells.

\section{Figure 2. CRISPR/Cas9-mediated gene editing of ex vivo T cells does not affect $T$ cell function} IFN $\gamma$, TNF $\alpha$, IL-2, IL-4, IL-5 and IL-10 release was evaluated using a cytokine bead array kit. (A,B) Electroporation with Cas9/IFNy gRNA RNP complexes results in reduced IFN $\gamma$ release whereas no differences are observed in the release of TNF $\alpha$ and IL-2, 2 days after electroporation. Atypical induction of IL-4, IL-5 or IL-10 are also not observed. (C) t-SNE plots showing the reduced IFNy release after stimulation with beads at $t=0$ and $t=4$ hours after electroporation. (D) IFN , TNF $\alpha$ and IL-2 cytokine-producing CD8 T cells after activation with anti-CD3/CD28 beads at 0, 30, 60, 120 and 240 minutes after RNP nucleofection, followed by 2 day culture and restimulation using PMA/ionomycin. (E) Scatterplot showing the anti-CD3/CD28 bead- or PHA-based expansion of T cells directly following electroporation over 14 days. (F) IFNY and IL-2 production of 14 days expanded gene-edited CD8 T cells. (G) Flow cytometry contour plot showing the TCR $\alpha \beta$ knockout in CD8 and CD4 T cells 48 hours after electroporation (G). (H) Expansion capacity of anti-CD3/CD28 activated TCR $\alpha \beta$ knockout cells over 14 days. (I) Flow cytometry contour plot left) and scatterplot (right) showing the TCR $\alpha \beta$ knockout in CD8 and CD4 T cells after 14 days.

\section{Figure 3. CRISPR/Cas9 gene editing preferentially targets memory T cells.} Gene editing of TCR $\alpha \beta$, IFNץ, CBLB or FAS in PBMCs. (A) Viability of CD8 versus CD4 TCR $\alpha \beta$ knockout T cells after in vitro stimulation with IL-2, IL-15 and/or IL-7 for eight days. (B) Percentages of CD8 and (C) CD4 memory T cells within TCR $\alpha \beta$ knockout T cells compared to TCR $\alpha \beta$-proficient cells after co- 
455 culturing with IL-2, IL-15 and/or IL-7 for eight days. (D) Percentages of CD8 memory and (E) CD8 naïve

456 T cells expressing TCR $\alpha \beta$ eight days after gene editing. (F) Gene editing efficiency of CD8 memory T

457 cells compared to CD8 naïve T cells. (G) Percentages of CD4 memory and (H) CD4 naïve T cells

458 expressing TCR $\alpha \beta$ eight days after gene editing of the TCR $\alpha \beta$ receptor. (I) Gene editing efficiency of

459 CD4 memory T cells compared to CD4 naïve T cells. Culture conditions as indicated.

460

461 Figure 4. CRISPR/Cas9 editing does not influence the antigen-specificity of T cells.

462 Gene editing of IFNY, CBLB or FAS in PBMCs. (A) Representative flow cytometry contour plots showing

463 the expression of T cell activation markers CD69 and CD137 after ex vivo and (B) post-IVS stimulation

464 with CEF. (C) Scatterplot representing the percentage of CD8 T cells expressing both activation

465 markers CD137 and CD69 within variable CRISPR gene editing conditions and untouched control cells

466 after ex vivo stimulation at day 2 and (D) post-IVS stimulation with and without CEF peptides at day 13.

467 Blue dots represent PBMCs not stimulated with CEF peptides and orange dots PBMCs stimulated with

468 CEF.

Supplementary figure 1. CRISPR/Cas9-mediated gene editing of ex vivo T cells does not affect T cell

471 function.

472 Scatterplots representing the IFNy-, IL-2, and TNF $\alpha$ producing CD8 and CD4 T cells after activation with

473 anti-CD3/CD28 beads, or PHA, 14 days after expansion (relates to figure 2f).

474

475 Supplementary figure 2. Gating strategy.

476 Scatterplots representative for the gating strategy used throughout the manuscript. The depicted

477 scatterplots show gene edited PBMCs after CBLB knockout and IVS stimulation with CEF. Cell

478 populations are depicted above the FACS images and the gated population are depicted below.

479

480

481

482 
bioRxiv preprint doi: https://doi.org/10.1101/2021.11.12.468355; this version posted November 13, 2021. The copyright holder for this

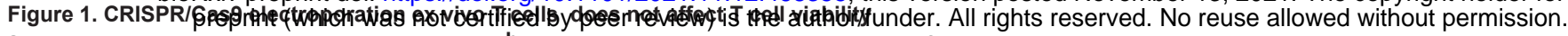
b

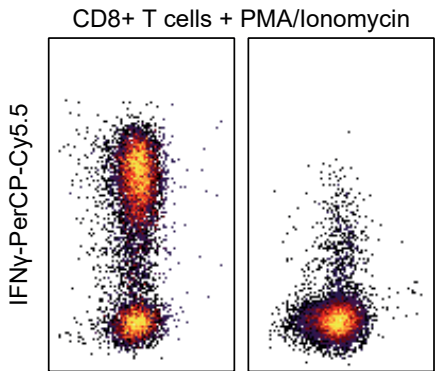

control

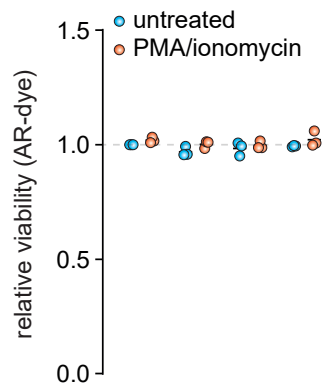

$\begin{array}{ccccc}\text { Electroporation } & - & + & + & + \\ \text { Cas9/tracRNA } & - & - & + & +\end{array}$

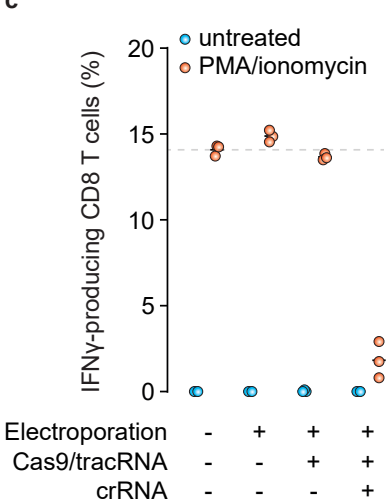

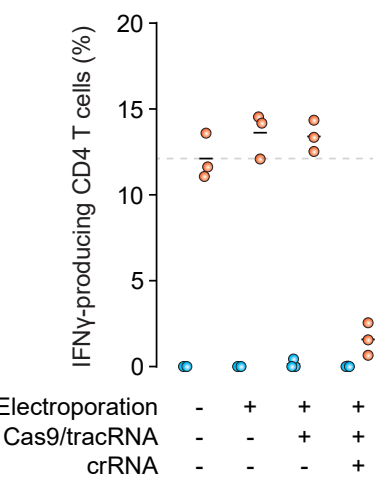

d

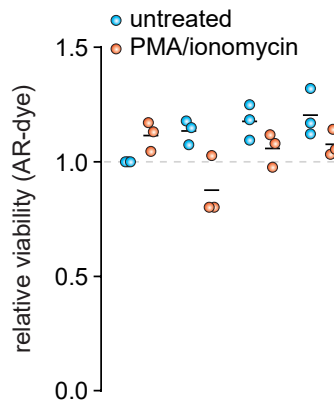

Cas9 $(\mu \mathrm{g} / \mathrm{mL}) \quad 0 \quad 100 \quad 250 \quad 500$ $100 \mu \mathrm{L}$ cuvette

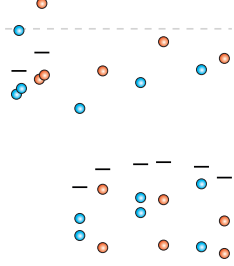

$0 \quad 100 \quad 250 \quad 500$ $20 \mu \mathrm{L}$ cuvette

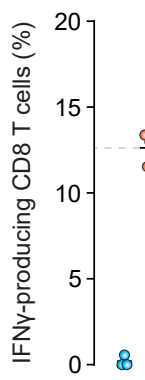

$\overline{0}^{\overline{0}} 0^{8} \overline{0}^{\circ}$

$0 \quad 100 \quad 250 \quad 500$ $100 \mu \mathrm{L}$ cuvette

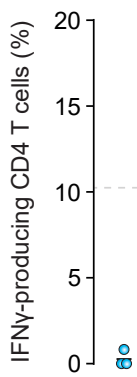

: $\overline{0} \overline{0} \div$ :

$0 \quad 100 \quad 250 \quad 500$

$20 \mu \mathrm{L}$ cuvette

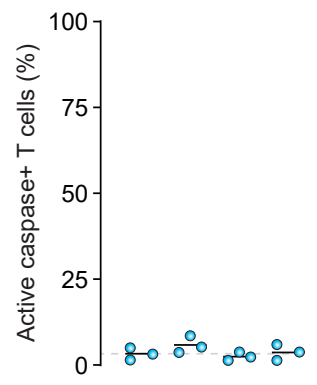

Electroporation - +++

Cas9/tracRNA - - + +
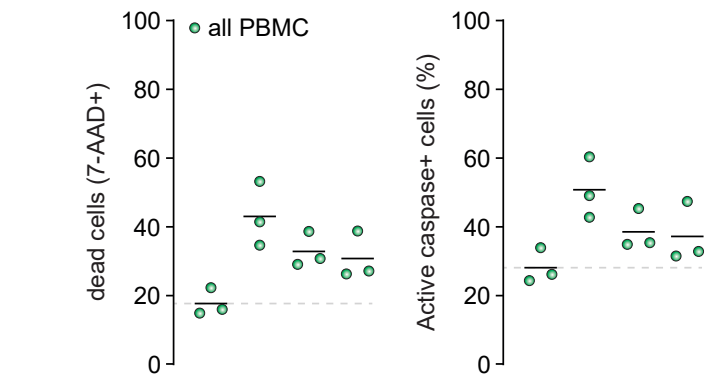
bioRxiv preprint doi: https://doi.org/10.1101/2021.11.12.468355; this version posted November 13, 2021. The copyright holder for this preprint (which was not certified by peer review) is the author/funder. All rights reserved. No reuse allowed without permission. Figure 2. CRISPR/Cas9-mediated gene editing of ex vivo T cells does not affect $T$ cell function
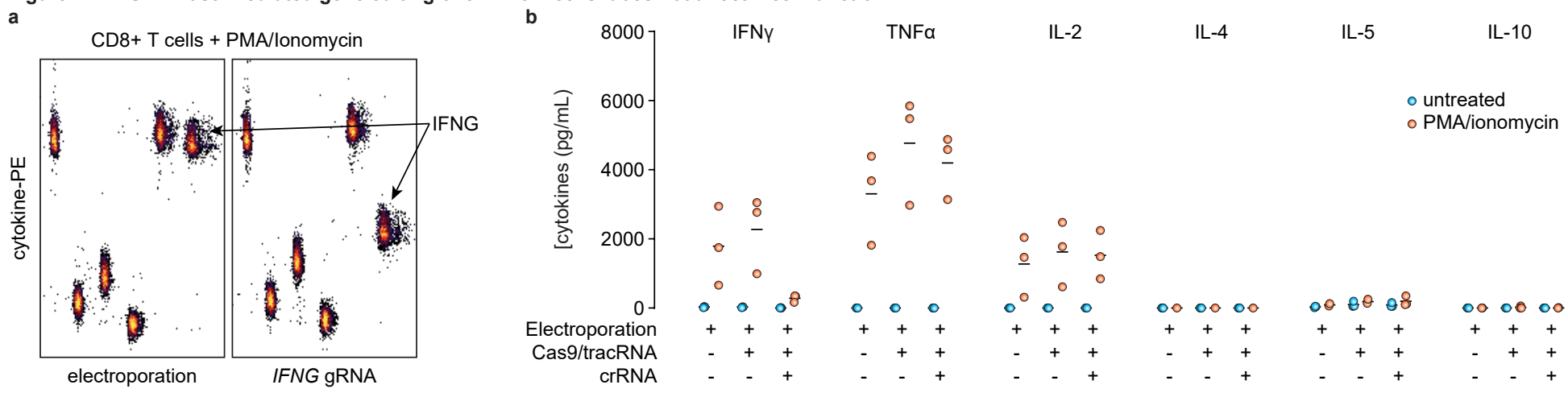

C

d
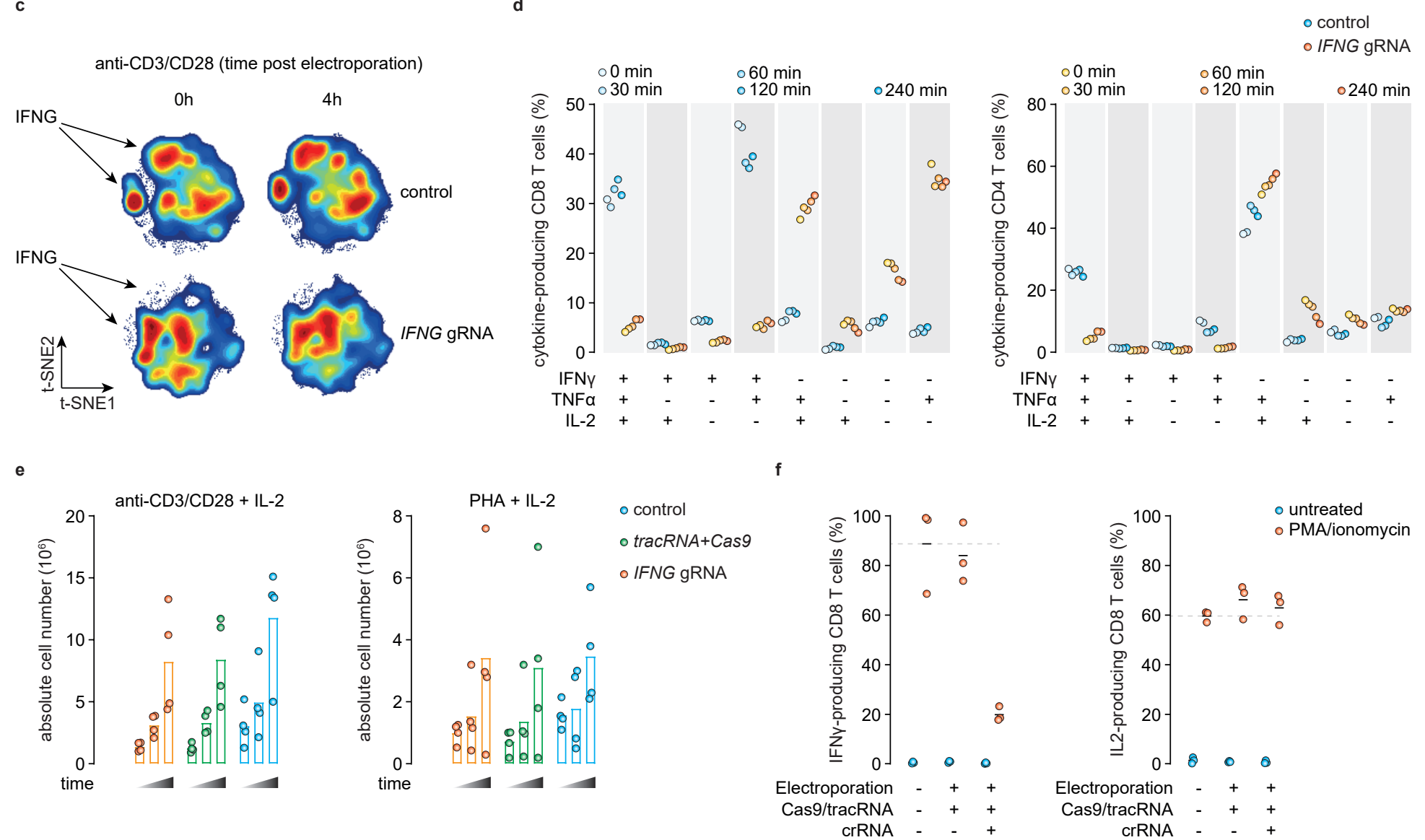

g
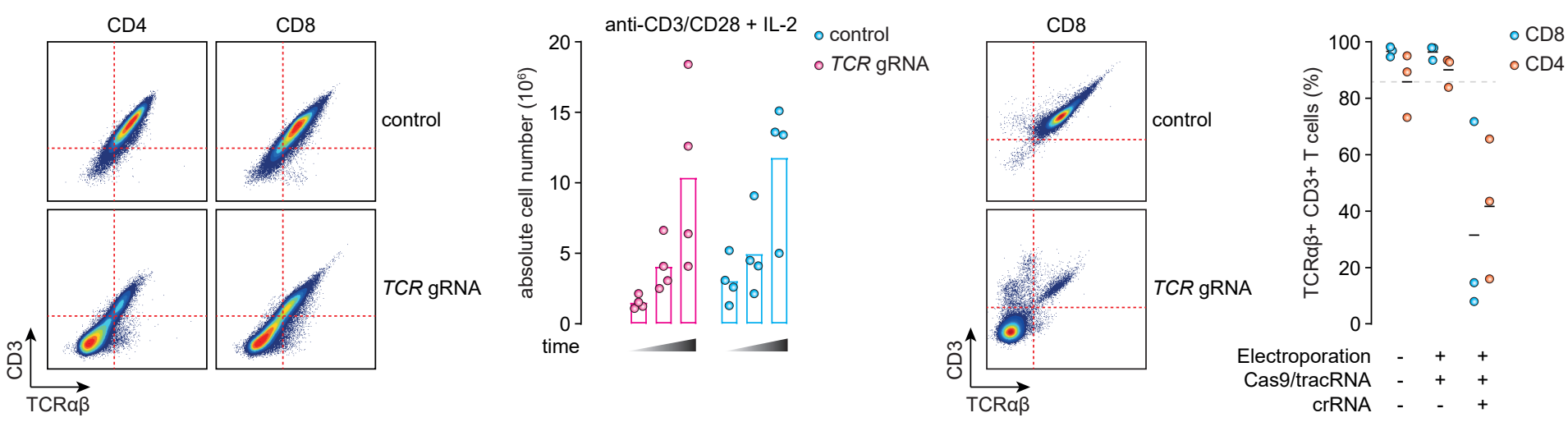
Figure 3. CRIS $\beta$ RikBiy preprint doi: https://doi.org/10.1101/2021 11.12.468355; this version posted November 13,2021 . The copyright holder for this

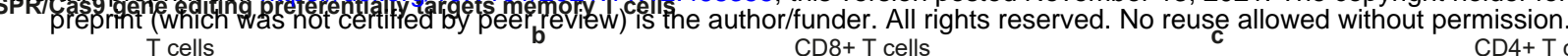
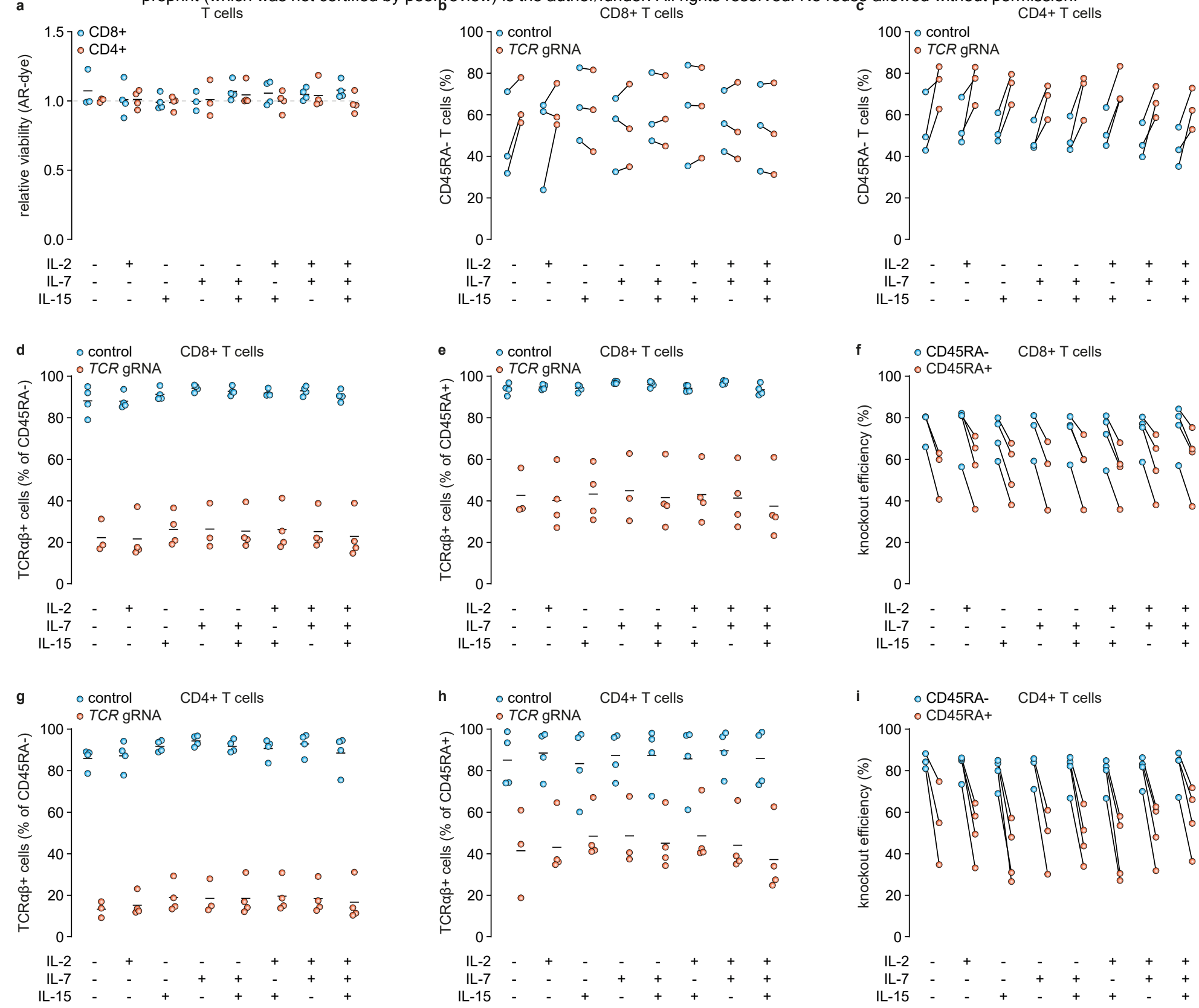
bioRxiv preprint doi: https://doi.org/10.1101/2021.11.12.468355; this version posted November 13, 2021. The copyright holder for this Figure 4. CRISPR Rreprint. (which was not fertified by peer reviewy is the authpr/funder. All rights reserved. No reuse allowed without permission.

ex vivo PBMC (CD8+ T cell gate)

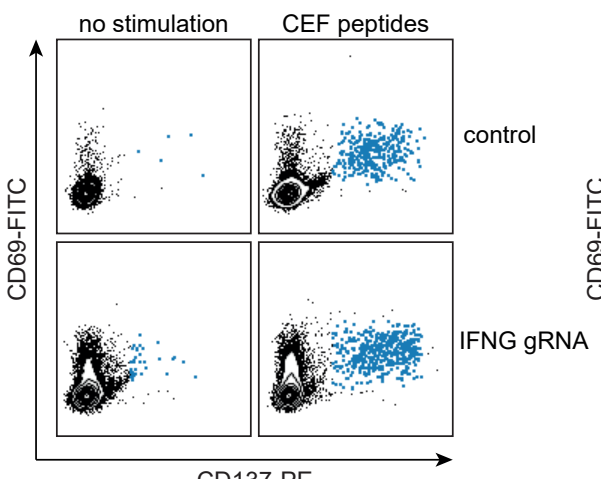

CD137-PE
post-IVS PBMC (CD8+ T cell gate)

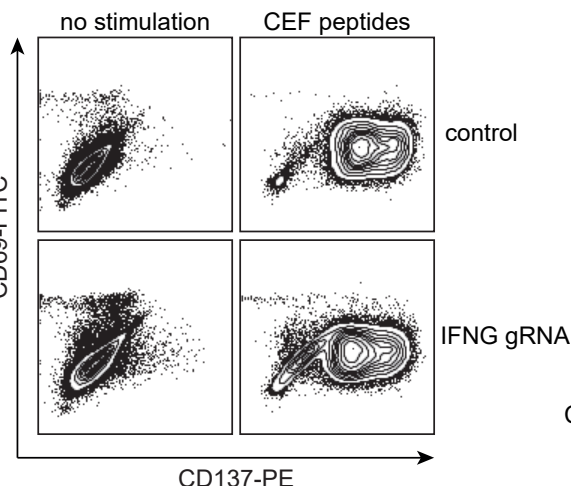

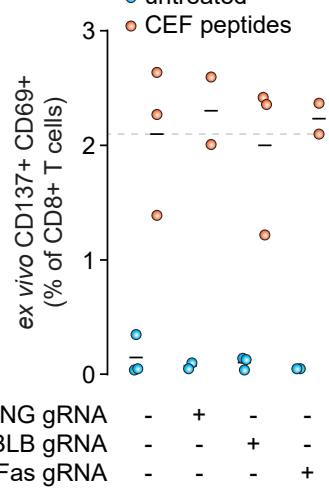

d

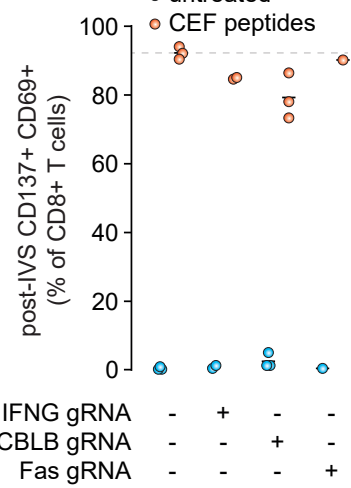

